\title{
PROGRAMA CON ARTISTAS EMERGENTES ${ }^{1}$
}

\section{Program of artistic interventions}

\author{
Clara Megías \\ Universidad Complutense de Madrid \\ claramegias@art.ucm.es \\ En colaboración con Eva Morales, RaQuel SACristán, \\ Claudia Claremi y Alma Orozco
}

Recibido: 3 de febrero de 2011

Aprobado: 6 de abril de 2011

\section{Resumen:}

El programa de intervenciones de creadores del panorama artístico emergente de Madrid en la Unidad de Psiquiatría Adolescente del Hospital Gregorio Marañón nace en verano de 2010. El equipo del proyecto curARTE pretende conectar a los pacientes de la Unidad con la vida cultural del la ciudad, ofrenciéndoles la oportunidad de participar en un proyecto artístico "real" cuyos resultados tendrán una repercusión más allá de las paredes del hospital, abriendo de este modo una ventana hacia el mundo exterior y hacia el futuro.

Palabras clave: adolescencia, Psiquiatría, artistas emergentes, participación, futuro.

Megías, C. Morales, E. Sacristán, R. Claremi, C. Orozco, A. 2011: Programa con artistas emergentes. Arte, Individuo y Sociedad, Vol. 23, Núm. Especial, 55-67.

\begin{abstract}
:
The program of artistic interventions in the Adolescent Psychiatric Unit of the Hospital Gregorio Marañón by a number of artists of the emerging art scene in Madrid started in summer 2010. CurARTE Project team attempts to connect patients of the Unit with the cultural life of the city, offering them the opportunity to participate in a "real" art project whose results woud impact beyond the hospital walls, opening a window to the outside world and to the future.
\end{abstract}

Key words: Teenagers, Psychiatry, emerging artist, participation, future.

\section{Sumario:}

1. Introducción. 2. Contexto. 3. Descripción del programa. 4. Conclusiones. Referencias.

\footnotetext{
${ }^{1}$ Esta investigación ha sido financiada por el Ministerio de Ciencia e Innovación (EDU20085441-C02-00/EDU)
} 


\section{Introducción}

Desde verano de 2010, Clara Megías (becaria FPU del proyecto curARTE) coordina un programa de intervenciones de creadores del panorama artístisco emergente de Madrid en la Unidad de Psiquiatría Adolescente del Hospital Gregorio Marañón. Con este programa no sólo se pretende dar a conocer a los pacientes de la Unidad la obra de artistas emergentes. El principal objetivo es que los pacientes formen parte de un proyecto artístico "real", cuyos resultados tendrán una repercusión más allá de las paredes del hospital.

\section{Contexto}

\subsection{Capa geográfica}

Todas las intervenciones que se describirán a continuación fueron diseñadas para la Unidad de Psiquiatría Adolescente del Hospital Gregorio Marañón:

La Unidad de Hospitalización Breve de Adolescentes del Hospital General Universitario Gregorio Marañón está ubicada dentro del Servicio de Psiquiatría de este hospital. Su objetivo es el estudio y estabilización de aquellos cuadros psiquiátricos agudos que requieran hospitalización a tiempo total y durante un periodo aproximado de una a tres semanas. Para ello cuenta con 20 camas y un personal multidisciplinar especializado en la atención a esta población. La Unidad de Adolescentes nació en noviembre del año 2000 y da en la actualidad cobertura a una población de aproximadamente 3.786.572 habitantes, correspondientes a las áreas sanitarias 1, 3, 4, 5, 6, 9 y 11 de la Comunidad de Madrid (web oficial del hospital: http://www.hggm.es/ua/)

En España, los mayores de 14 años suelen ser hospitalizados con los pacientes adultos, aunque en algunos casos se contempla la hospitalización en planta pediátrica hasta los 16 años, con una atmósfera más adecuada pero igualmente insuficiente. La Unidad de Psiquiatría Adolescente del Hospital Gregorio Marañón es uno de los pocos casos de espacios hospitalarios de uso exclusivo para adolescente que hemos encontrado. Cuenta con una sala multiusos dotada de mobiliario móvil, sillones, armarios con materiales cerrados con llave y un televisor con DVD.

\subsection{Capa humana}

Independientemente del tipo de hospitalización, el proceso de construcción de la identidad se ve gravemente obstaculizado cuando el adolescente sufre alguna enfermedad y debe permanecer hospitalizado. Por esta razón, para diseñar una propuesta educativa para adolescentes en el contexto la Unidad de Psiquiatría Adolescente debemos tener en cuenta los siguientes factores (Pascale y Ávila, 2007):

- Desarrollo cognitivo: el aislamiento y la escolaridad discontinua en caso de enfermedades crónicas puede producir un retraso en el desarrollo cognitivo y social. El tránsito por la adolescencia es aún más complejo en pacientes que sufren trastornos psiquiátricos. 
- Planes de futuro: durante la hospitalización los planes de futuro se ven paralizados y el sentimiento de estar viviendo un periodo de moratoria se acentúa.

- Comportamiento antisocial: algunos de los pacientes de la unidad pueden haber sido ingresados debido a una conducta violenta o antisocial. Por ello hay que tener en cuenta un leve factor de riesgo y actuar con rapidez avisando al personal sanitario cuando se produzca un conflicto entre pacientes.

- Aislamiento: cualquier propuesta que suponga una conexión con el mundo fuera del hospital es bien recibida y supone gran motivación, debido a que el contacto con el exterior se encuentra controlado (los pacientes no tienen acceso a Internet y la única ventana que tienen al exterior es la televisión, que se visiona de manera colectiva en la sala multiusos).

\section{Descripción del programa}

\subsection{Metas generales}

Las metas del programa de intervenciones de artistas emergentes se proyectan en una doble dirección:

- Poner al alcance de los pacientes hospitalizados procesos de producción artística emergentes.

- Dar a los artistas la oportunidad de conocer un realidad a la que no tendrían acceso de otra manera.

\subsection{Sesiones}

El programa está compuesto por las intervenciones de cinco artistas, todas ellas mujeres que trabajan en el ámbito de la acción artística y el arte contextual. Todas las artistas entienden el arte como un lugar para relacionarse y reflexionar sobre las problemáticas de nuestra sociedad. Los proyectos desarrollados en este programa son propuestas de intervención en el espacio del hospital y sus alrededores, reivindicando de este modo la recuperación del espacio público por parte de los ciudadanos y su uso creativo y responsable.

\section{Área de salud con Raquel Sacristán}

\section{Bio}

Raquel Sacristán (www.sakristan.com) es una creadora multidisciplinar especializada en Artes Visuales por la Universidad Complutense de Madrid. Sus trabajos tienen el objetivo de eliminar las barreras entre códigos estéticos, lenguajes artísticos y diferentes medios de expresión. Actualmente su trabajo se centra en catalizar procesos creativos en los que la obra surge del encuentro y la colaboración directa con personas o comunidades que, en principio, no se sienten cercanos al contexto del arte contemporáneo. Combina lo más primitivo del arte con la crítica social, y para ello utiliza diferentes soportes y entornos para expresarse, dando prioridad a los espacios públicos que están especialmente degradados. 


\section{Sinopsis del proyecto}

El objetivo de este proyecto es crear una pieza de arte urbano (pensada para ser instalada en la calle), con adolescentes que no pueden salir del Hospital por un periodo de tiempo, y así reflexionar sobre cómo transformar los espacios públicos en zonas más saludables, en espacios dentro de la ciudad que nos hagan sentir mejor. Para ello artista, educadores y pacientes trabajaron juntos en la creación de una instalación que sería un "Área de Salud" situada en la calle cerca del hospital Gregorio Marañón de Madrid, para que cualquier persona pudiera disfrutar de ella y, de este modo tratar de desdibujar las fronteras entre el interior y el exterior del espacio en que se encontraban los pacientes, reflexionando sobre el concepto salud y la incidencia del entorno en la misma.

\section{Ficha técnica}

Fecha

Duración

Participantes

Educadores
26 de julio de 2010

En la Unidad: 1h 30

Fuera de la Unidad: 1 h 30

Tres chicos y una chica

Víctor Nieto, Álvaro Valls

Metas educativas

- Entender que el arte urbano es algo más que el grafiti.

- Descubrir un tipo de arte fuera del mercado, que tiene como objetivo mejorar de forma creativa el espacio urbano.

- Entender el arte como un proceso performativo.

- Descubrir que el arte puede servir para mejorar hacer más saludable nuestro entorno.

\section{Proceso}

En la Unidad (con los pacientes):

1. Presentación de obras de artistas urbanos que no utilizan el graffiti de forma destructiva, entre ellos, la propia artista invitada.

2. Creación de los elementos que formarán parte de la instalación: en primer lugar, transplantar plantas de hierbabuena usando mascarillas como macetero colgante (Fig. 1 y 2) y en segundo lugar, escribir mensajes optimistas para compartir con los viandantes. Estos mensajes irán colgados junto con las plantas.

En la calle (la artista con el equipo de educadores):

3. Buscar un lugar donde realizar la instalación. Para ello son necesarios dos árboles próximos (Fig. 3). 
4. Colgar una hamaca entre los dos árboles y sobre ella una cuerda de la que penderán las macetas/mascarilla, un cartel con el título ÁREA DE SALUD y los mensajes (Fig.4).

Después de la instalación la artista vuelve a la unidad hospitalaria para mostrar a los pacientes fotografías de todo el proceso.
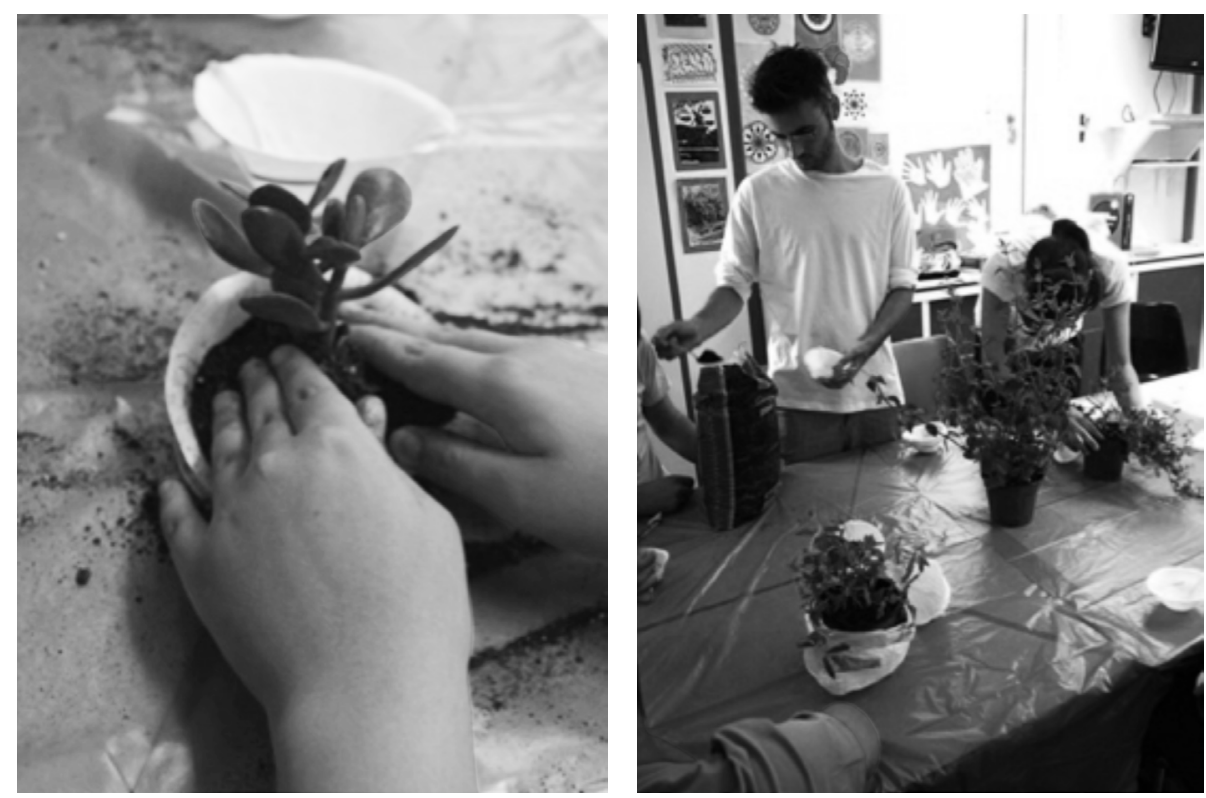

Fig.1.y 2.. Fotografías tomadas dentro de la Unidad.

\section{Certificados de alta con Claudia Claremi}

\section{Bio}

Claudia Claremi (www.claremi.blogspot.com) tras estudiar Bellas Artes en Londres y ampliar sus estudios en La Habana, vive actualmente en Madrid, donde desarrolla su obra artística en la que investiga el valor no material de lo material. Una parte de sus proyectos se basa en la entrega de regalos y el estudio de las posibilidades de este gesto. En otros, utiliza estructuras consolidadas en el sistema de mercado o la Administración pública para crear experiencias íntimas.

\section{Sinopsis}

A lo largo de la vida acumulamos involuntariamente certificados que marcan acontecimientos y momentos vitales de cada uno. En este proyecto los adolescentes ingresados elaboraron su propio certificado de alta médica. Cada uno decidió su día de salida del hospital y creó el documento con los elementos necesarios (sello, firma, cuerpo de texto...), usándolos como herramientas plásticas. En este proyecto el cer- 
tificado es cuestionado como expresión de autoridad, pero sin abordar los conceptos de falsificación y autenticidad. Los participantes deciden sobre su propia situación y asumen, desde el propio hospital, el rol incuestionable de quien expide un certificado.

\section{Ficha técnica}

Fecha

Duración

Participantes

Educadores
16 de agosto de 2010 y 5 de marzo de 2011 $1 \mathrm{~h} 30$

3 en la primera edición, 9 en la segunda

Víctor Nieto, María José Martínez y María Chacón

Metas educativas

- Reflexionar sobre el uso y la existencia de los certificados.

- Entender el arte como un proceso performativo.

\section{Proceso}

Detonante:

$\mathrm{Al}$ entrar en el espacio de trabajo los participantes se encuentran a la artista vestida de secretaria (Fig. 5), escribiendo a máquina en un escritorio dotado con material de oficina variado: máquina de escribir, sellos de distintos tipos, un bote con bolígrafos, carpetas y archivadores, una pequeña planta y un el título universitario de la artista enmarcado. La escena provoca la curiosidad de los participantes, que comienzan a hacer preguntas. De este modo la artista introduce la temática de los certificados y enseña los que ha ido coleccionando.
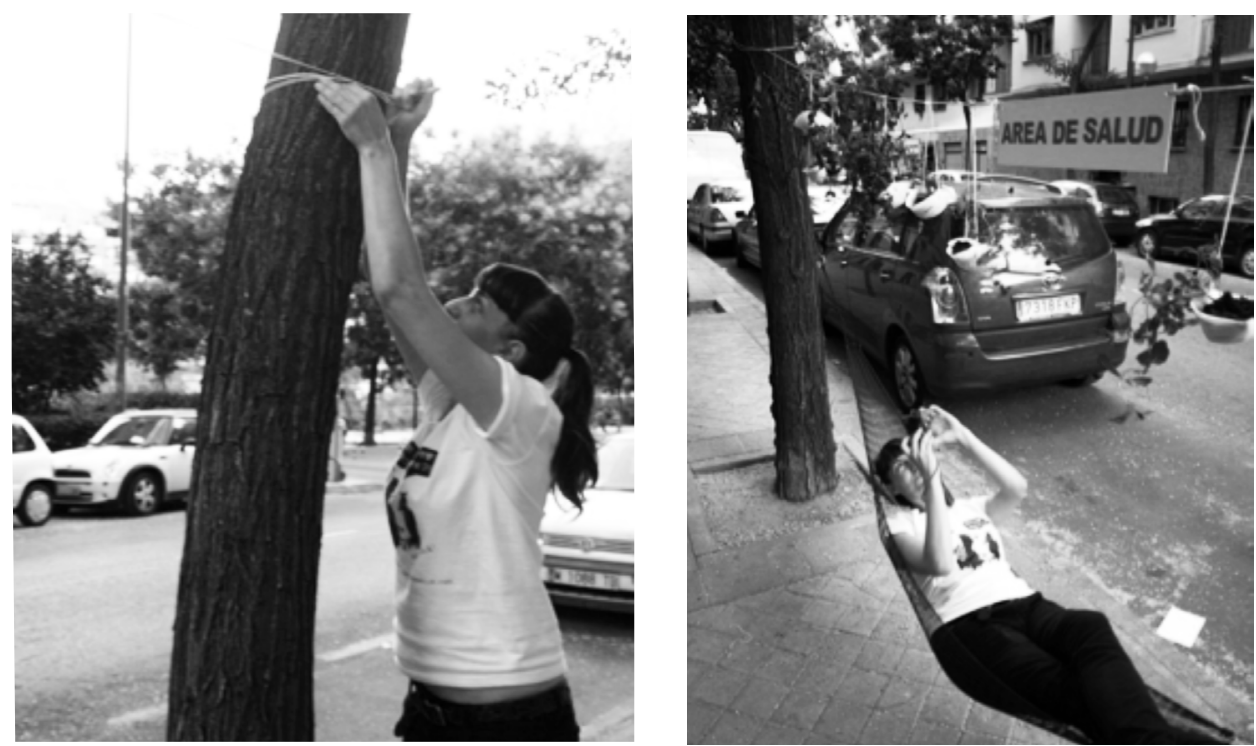

Fig.3,4. Fotografías tomadas fuera de la Unidad. 


\section{Acción:}

Los participantes pasan a diseñar su propio certificado empleando los materiales antes citados. Para esta experiencia se contó con 6 máquinas de escribir (Fig. 5, 6 y 7).
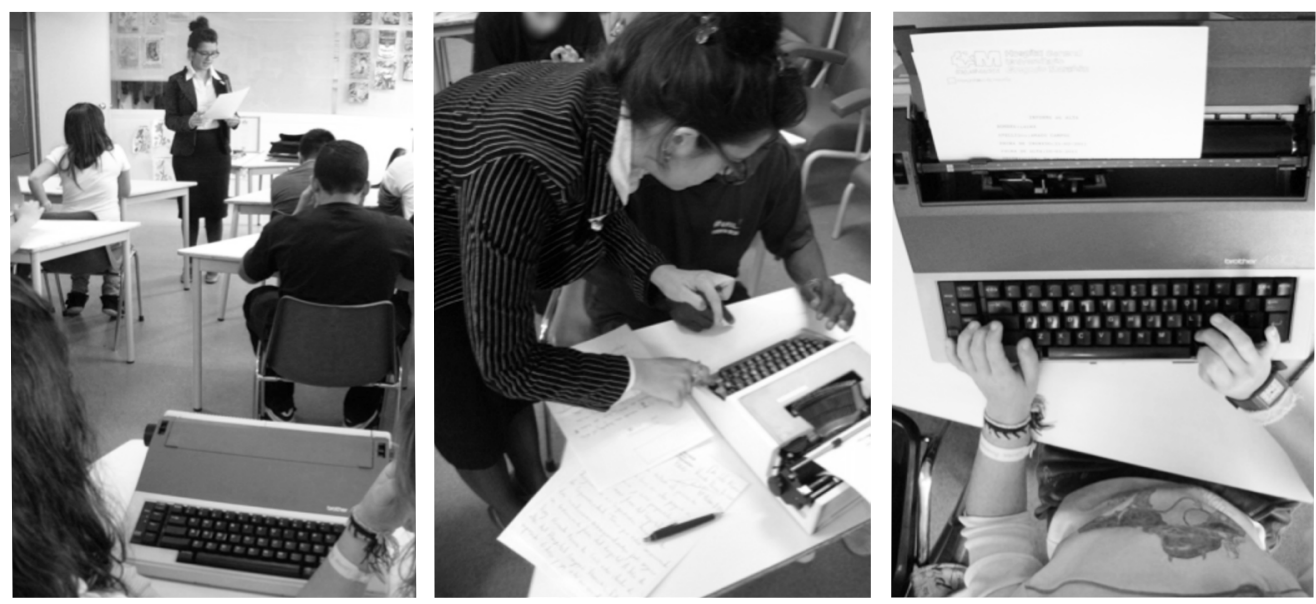

Fig.5,6,7. Fotografías tomadas durante el taller Certificados de Alta.

\section{Tormenta con Colectivo Núbol}

\section{Bio}

Núbol (www.nubol.net) es un colectivo formado por Clara Megías y Eva Morales, que cree en la necesidad urgente de incorporar la creación artística actual en cualquier contexto. Cree en un arte actual transversal y multidisciplinar como el mundo en el que vivimos. Núbol trabaja desde la experiencia colectiva creando proyectos entre lo artístico y lo educativo. Entre sus proyectos cuenta con acciones en red y talleres en distintos contextos (hospitales, universidades, museos).

\section{Sinopsis}

A partir del concepto de tormenta de ideas, el Colectivo Núbol creará dos tormentas visuales con el objetivo de entender el arte como un medio para reflexionar sobre el mundo en el que vivimos y como una manera de recapacitar sobre uno mismo. ¿Quién es el poseedor de una idea? Las ideas habitan en el cerebro, al igual que las nubes el cielo. Ni las unas ni las otras se pueden palpar. Se escapan de los límites físicos. Son libres, etéreas, transmutables, efímeras. Se pueden enlazar con otras o simplemente desaparecer.

\section{Ficha técnica}

Fecha

Duración
12 de mayo de 2010

2 horas 

Participantes
Cuatro chicas y siete chicos
Educadores
Las propias artistas

Metas educativas

- Entender que el arte puede ser una manera de reflexionar sobre el mundo.

- Descubrir que el arte es una manera para conocerse a uno mejor.

- Comprender que lo importante en el arte es el proceso.

- Descubrir las ventajas del trabajo colaborativo.

\section{Proceso}

Detonante:

Los pacientes dibujan sobre un folio blanco una silueta humana, le dan la vuelta y dibujan una nube detrás de la zona donde está la cabeza de la silueta. Al levantar el folio y mirarlo al trasluz se puede ver la nube dentro de la cabeza, como si se tratara del cerebro del personaje. Esta es una manera de introducir el concepto central del Colectivo Núbol, extraído de la obra de Juan Zamora en la que las nubes/cerebro representan la intangibilidad del mundo de las ideas.

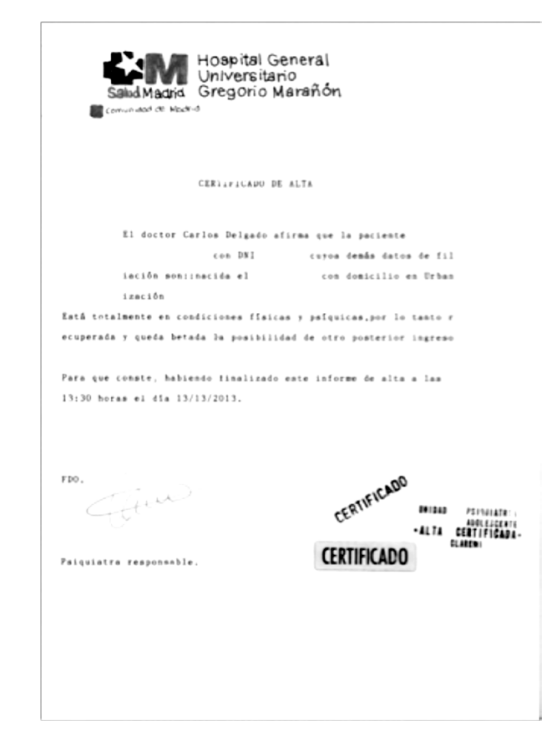

Fig.8. Certificado de alta resultado del taller.

\section{Acción 1:}

Con la TORMENTA MUNDIAL, creada a partir de periódicos viejos, los participantes opinan sobre la situación actual del mundo, seleccionando aquellos fragmentos de los periódicos que representen esta situación y para después transformarlos en nubes (Fig. 9). 


\section{Acción 2:}

Con la TORMENTA VITAL, creada a partir de fotografías de nubes realizadas por Núbol, los pacientes escriben mensajes que les gustaría compartir con los demás (Fig. 10). Una vez escritos los mensajes, se unen todas las fotografías creando un panel que será instalado en la pared de la unidad (Fig. 11)

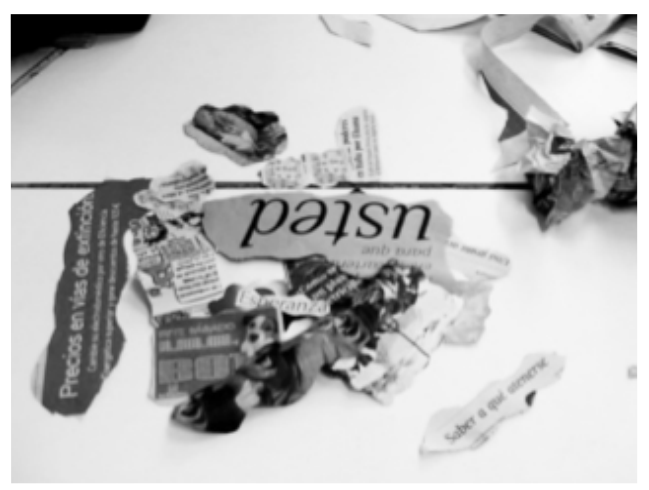

Fig.9. Fotografía de la Tormenta Mundial.

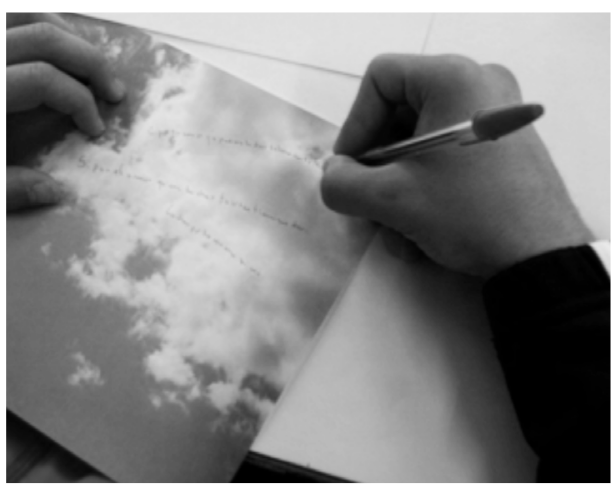

Fig.10. Fotografías del proceso de realización de la Tormenta Vital.

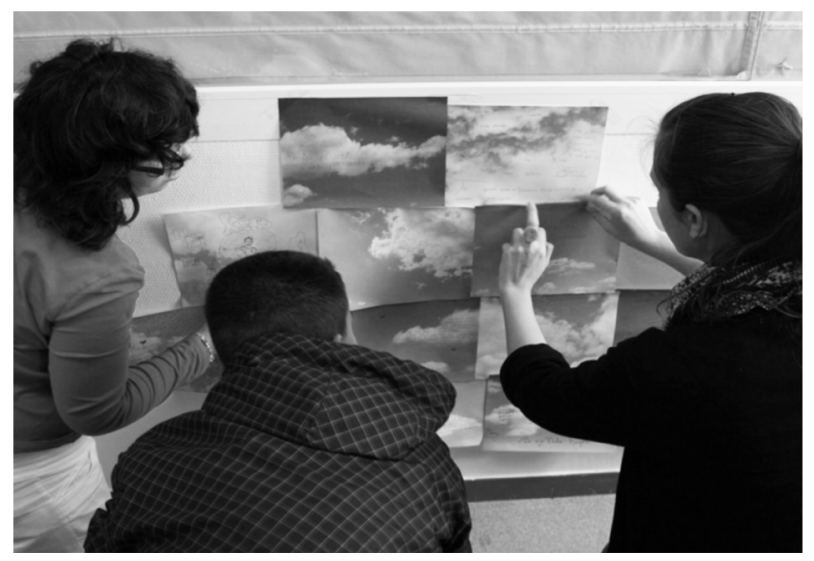

Fig.11. Fotografías del proceso de realización de la Tormenta Vital.

\section{Te voy a hacer un monumento con Alma Orozco}

\section{Bio}

Alma Orozco (almaorozco.blogspot.com) es creadora multidisciplinar y comunicadora social. Muy preocupada por los mecanismos que afectan a la construcción de la realidad, trabaja la creación de imaginarios sociales a través del arte, desarrollando la construcción de espacios de libertad creativa. Asimismo, trata de democratizar las prácticas artísticas, y trabaja como facilitadora de arte con niños, personas con discapacidad y desarrollando proyectos de arte entendido como herramienta para hacer ver en la sociedad de la sobreexposición mediática de la mirada. 


\section{Sinopsis}

En este proyecto se trabajó sobre la construcción identitaria a través de la inversión de la idea de monumentalidad. Se trata de, en lugar de construir un monumento a partir de una identidad, construir una identidad a partir de un monumento. Pensar qué parte de nosotros mismos nos gustaría que estuviera en lo representado y, a partir de ahí, construir una identidad grupal compuesta de los fragmentos que conforman las identidades individuales. Utilizando el objeto escultórico y figurativo como recurso, se construyó toda la historia ausente que hay detrás del mismo.

\section{Ficha técnica}

Fecha

Duración

Participantes

Educadores
19 de marzo de 2011

1 h 30

Cuatro chicas y siete chicos

Clara Doblar, Cristina Gómez, Clara Megías y

Mónica Sáinz

Metas educativas

- Reflexionar sobre la existencia de monumentos y su significado.

- Ser capaz de trabajar en grupo y llegar a un consenso.

- Valorar el proceso como verdadero objetivo del acto artístico.

\section{Proceso}

Detonante:

Se coloca en medio de la sala un monumento en blanco, una efigie humana sin identidad y se comienza a reflexionar sobre los monumentos y su función.

Diseño de propuestas de intervención:

Se propone a los participantes el reto de dotar de identidad y significado a la escultura. Cada uno realiza un boceto de una posible intervención en el monumento. Al final se decide por medio de consenso qué intervención se va a realizar. En este caso, los participantes decidieron realizar un acto de "vandalismo" convirtiendo la escultura en un muñeco de vodú gigante. El muñeco representaría "su yo pasado", al cual renuncian mediante la sátira.

Intervención:

El grupo interviene la escultura conforme a lo pactado. Una vez terminada la intervención, el monumento será fotografiado en distintos puntos de la ciudad y todo el proceso se podrá seguir en el blog www.tevoyahacerunmonumento.wordpress.com 


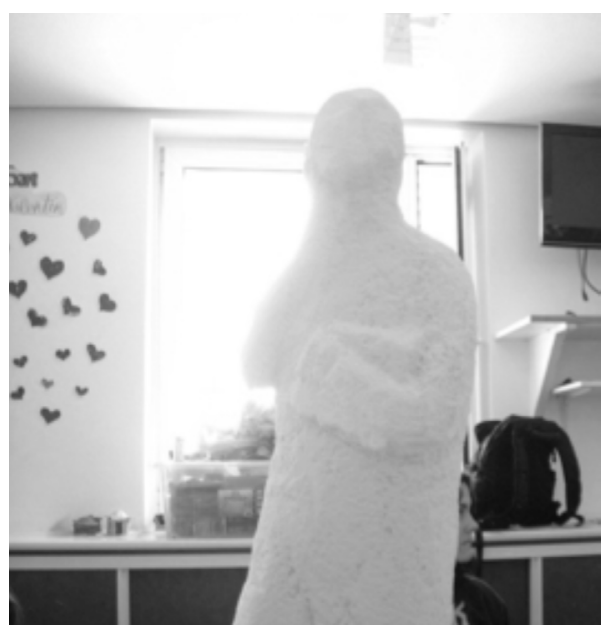

Fig.12. El monumento antes de intervención.

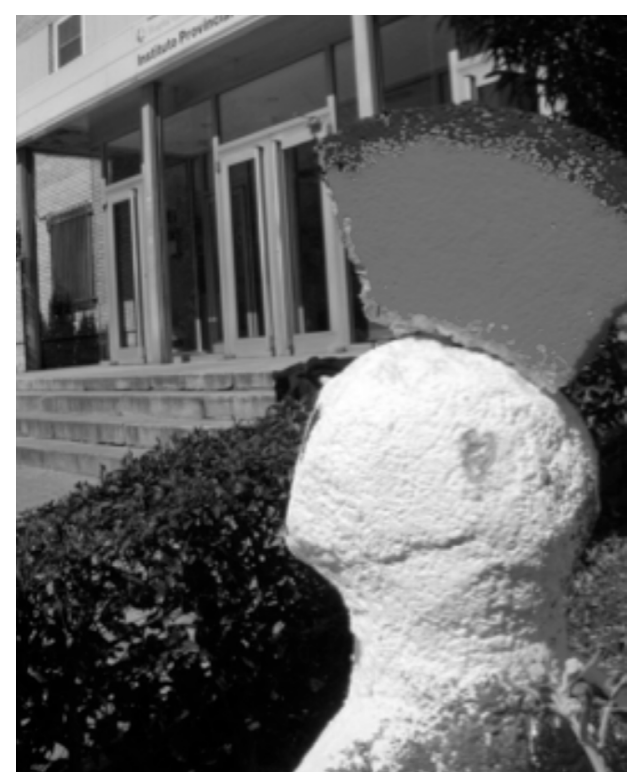

Fig.13. El monumento en el exterior del hospital, después de la intervención.

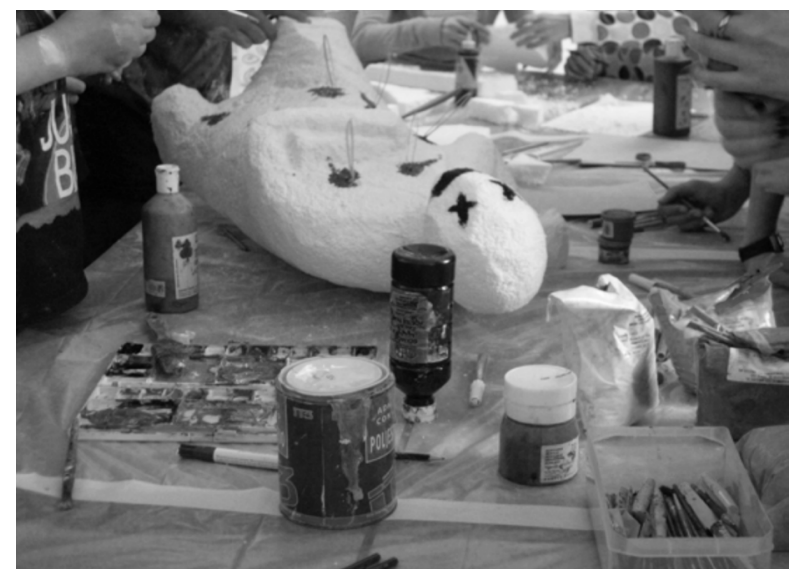

Fig.14. Fotografía tomada durante el proceso de intervención. 


\section{Conclusiones}

Como comentamos anteriormente este programa fue diseñado teniendo en cuenta los siguientes objetivos:

En primer lugar, poner al alcance de los pacientes hospitalizados procesos de producción artística emergentes. Invitar a los pacientes de la unidad a participar en proyectos "reales" de artístas en activo, proyectos que no se limitan a experiencias aisladas sino que tienen continuidad fuera del hospital es una metodología que presenta numerosas ventajas a la hora de trabajar con los pacientes de la Unidad de Psiquiatría Adolescente:

- Conexión con el exterior: la situación de angustia que produce el aislamiento en el que se encuentran los pacientes se ve aliviada al poder comunicarse, mediante la creación artística, con personas que no se encuentran en el hospital. Participar en un proyecto supone un acto de generosidad al crear una obra para otras personas.

- Continuidad en el futuro: al tratarse de proyectos que tienen una continuidad, los pacientes para quienes el futuro se plantea difícil e incierto, pueden escapar momentáneamente de su situación presente, formando parte de un proyecto que servirá para que otras personas más adelante puedan reflexionar a partir de sus creaciones.

- El arte como un lugar de encuentro: los proyectos que hemos descrito hacen especial hincapié en un tipo de acción artística enfocada en el proceso y no en el producto. El carácter performativo de estas intervenciones subraya las ventajas del trabajo colaborativo y su valor educativo.

- El arte como reflexión sobre su situación actual: todos los proyectos cuentan con un componente individual y autoreflexivo. De este modo los pacientes encuentran en cada proyecto un espacio íntimo en el que poder explorar sus ideas personales para más adelantes compartirlas.

- El arte como un modo de ciudadanía responsable: dado que muchos de los pacientes de la unidad han sido hospitalizados por problemas graves de conducta, es necesario realizar programas educativos que sirvan para desarrollar el sentido de la responsabilidad. Este programa ha reunido una serie de proyectos que invitan a un uso reponsable y reflexivo del espacio público.

Y en segundo lugar, no debemos olvidar que este programa también ha servido para dar a estas artistas la oportunidad de conocer un realidad a la que no tendrían acceso de otra manera. Las artistas participantes consideraron un reto diseñar una acción que supusiera una "vía de escape que ayude a dar salida a la angustia que pueden causar los límites a los que estas personas se enfrentan" según palabras de una de las participantes. Alma Orozco, después de su intervención, comentó lo siguiente:

De esta manera, es casi una responsabilidad que la obra tenga una forma que mire hacia afuera y que no quede dentro de sí misma. Pienso que el arte es muy tautológico en este sentido y realizar este tipo de proyectos ayuda a colocarse en una disposición más abierta que la habitual dentro del contexto artístico.

Por otro lado, Claudia Claremi señaló que esta experiencia había sido todo un privilegio al poder conocer una realidad nueva. Al terminar su sesión nos comentó que 
el desconocimiento y la inseguridad inicial se había ido transformando, hasta el punto de llegar a ver el ambiente del hospital como algo familiar. Este acercamiento se produce en los dos sentidos: a lo largo de dos horas el artista se familiariza con el lugar de la misma forma que los ingresados se acercan al arte conectándolo con el exterior.

\section{Referencias}

Pascale, P. y Avila, N. 2007: Una experiencia de creatividad con adolescentes hospitalizados: unidad de psiquiatría de adolescentes del Gregorio Marañón. Arte, individuo y sociedad, 19, 207-246.

\section{WEB}

www.almaorozco.blogspot.com

www.nubol.net

www.claremi.blogspot.com

www.sakristan.com 